\title{
Triple-synergistic 2D material-based dual-delivery antibiotic platform
}

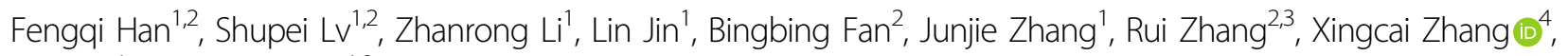
Lei $\operatorname{Han}^{1}$ and Jingguo $\mathrm{Li}^{1,2}$

\begin{abstract}
Two-dimensional (2D) nanomaterials have raised significant interest in not only energy and environmental fields but also biomedical areas. Among these materials, one type that has many interesting properties and possesses numerous exciting applications is graphene oxide (GO)-based 2D materials. However, their poor stability in aqueous solutions and weak bioactivities limit their use in biomedical applications, especially antimicrobial fields. In this study, GO was functionalized with hydrophilic polymers and used as a vector for silver nanoparticles (Ag NPs) and sulfadiazine (SD). The stability of the material in aqueous solutions was greatly improved. The antibacterial activity of the novel hybrid antibacterial system (HAS) was enhanced by over 3 times compared to that of the system lacking SD. The antibacterial performance of the HAS was due to the triple synergy: bacterial capping, puncture, and inhibition. This study provides new insights into the design and fabrication of surface-modified $\mathrm{GO}$ and carbon materials and their 2D hybrid multifunctional materials for advanced applications including biomedical and especially antibacterial applications, broadening the design and application scope of carbon and 2D materials.
\end{abstract}

\section{Introduction}

Graphene oxide (GO), an important two-dimensional (2D) nanomaterial, has attracted extensive attention due to its unique physical, chemical, and biological properties $^{1,2}$. GO has a high specific surface area and has many carboxylic groups at its edges ${ }^{3,4}$. Since graphene was discovered in $2004^{5}$, its applications in various medical fields have been explored, including in photothermal therapy $^{6}$, bioassays ${ }^{7}$, and drug delivery ${ }^{8,9}$, as well as in antibacterial agents ${ }^{10}$. Recent research has shown that graphene-based materials have low cytotoxicity and exhibit antibacterial activity ${ }^{11-13}$. However, the antibacterial properties and biomedical applications of these materials have been limited due to their low solubility in aqueous solution ${ }^{14}$.

Correspondence: Bingbing Fan (fanbingbing@zzu.edu.cn) or

Xingcai Zhang (xingcai@seas.harvard.edu) or Jingguo Li (lijingguo@zzu.edu.cn)

'Zhengzhou University People's Hospital, Henan Provincial People's Hospital, Zhengzhou 450003, PR China

${ }^{2}$ School of Materials Science and Engineering, Zhengzhou University, Zhengzhou 450001, PR China

Full list of author information is available at the end of the article.
Silver (Ag) has been an efficient antibacterial agent for centuries. With the development of nanotechnology, silver nanoparticles (Ag NPs) have shown better antibacterial performance than traditional bulk silver. Recent studies have shown that smaller Ag NPs have a better antimicrobial effect ${ }^{15}$. However, small Ag NPs coagulate easily, limiting their biomedical applications. GO was chosen for loading Ag NPs because it has a high specific surface area and unique physicochemical properties.

Silver sulfadiazine (SSD) and sulfadiazine (SD) are broad-spectrum antimicrobial agents with wide clinical applications ${ }^{16}$. They are widely used in the clinical treatment of burn diseases and show an excellent treatment effect. We introduced SD and Ag NPs onto the surface of GO to improve the antimicrobial activity of the hybrid materials, taking advantage of the high specific surface area of GO.

Recently, Ag/rGO was successfully synthesized. However, the material has poor aqueous stability, and its small number of functional groups available for conjugating 


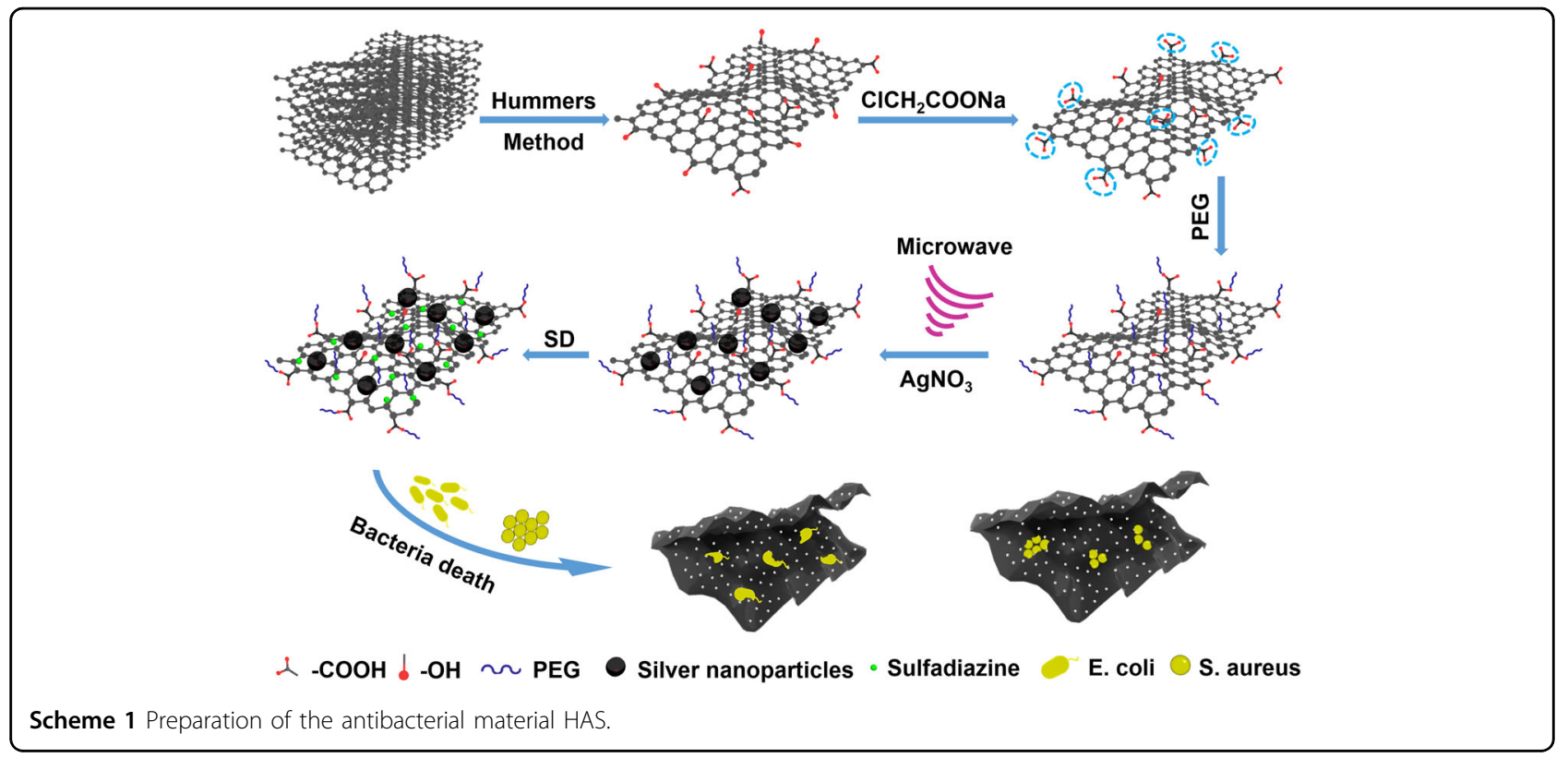

efficient macromolecule agents has greatly limited its applications $^{17,18}$.

In this study, hydrophilic macromolecules were introduced into carboxylated graphene oxide to improve the aqueous stability of the hybrid materials. To further enhance the antimicrobial effect, GO was surfacemodified as a vector to allow the loading of Ag NPs and SD. The facilely prepared 2D hybrid materials showed a good synergistic antibacterial effect.

\section{Experiments}

Materials

Graphite flakes were the product of Hengdeli Company (Qingdao, China). Sulfuric acid (98\%), $\mathrm{NaNO}_{3}, \mathrm{KMnO}_{4}$, $\mathrm{H}_{2} \mathrm{O}_{2}$ (30\%), silver nitrate (99.8\%), $\mathrm{HCl}$ (37\%), sodium hydroxide $(\mathrm{NaOH}), \quad \mathrm{N}, \mathrm{N}^{\prime}$-dicyclohexylcarbodiimide (DCC), 4-dimethylaminopyridine (DMAP), N,N-dimethylformamide (DMF, 99.8\%) and dimethyl sulfoxide (DMSO) were provided by J\&K Scientific Ltd. (Beijing, China). Sodium chloroacetate $\left(\mathrm{ClCH}_{2} \mathrm{COONa}\right)$ and polyethylene glycol (PEG-OH, Mn = 800) were provided by McLean Biochemical Technology Co., Ltd. (Shanghai, China). Sulfadiazine (SD) and silver sulfadiazine (SSD) were provided by Meilun Biochemical Technology Co., Ltd. (Dalian, China). Dialysis bags (Mw cut-off: $3.5 \mathrm{kDa}$ ) were purchased from Green Bird Technology Development Co., Ltd. (Shanghai, China). 3-(4,5-Dimethylthiazol2-yl)-2,5-diphenyl tetrazolium bromide (MTT), propidium iodide (PI) and 4',6-diamidino-2-phenylindole (DAPI) were obtained from Sigma Biochemical Technology Co., Ltd. (Shanghai, China). All chemical reagents were commercially available and were used without further purification. The deionized water used in the experiments was produced by an ultrapure water purification system.

\section{Synthesis of 2D antibacterial nanomaterials}

As shown in Scheme 1, polyethylene glycol-modified carboxylated graphene oxide was prepared as a carrier and release platform for Ag NPs and SD to build up the novel hybrid antibacterial system (HAS).

\section{Synthesis of carboxylated graphene oxide (GO-COOH)}

GO was prepared by the modified Hummers method with some modifications ${ }^{19}$. To synthesize carboxylated graphene oxide, GO suspension solution $(1 \mathrm{mg} / \mathrm{mL})$ was sonicated for $30 \mathrm{~min}$, and then, $\mathrm{ClCH}_{2} \mathrm{COONa}(1.2 \mathrm{~g}$, $40 \mathrm{~mL})$ was mixed with $\mathrm{GO}$ solution $(1 \mathrm{mg} / \mathrm{mL}, 20 \mathrm{~mL})$ under ultrasonication for $30 \mathrm{~min}$. Finally, sodium hydroxide solution $(10 \mathrm{~mol} / \mathrm{mL}, 40 \mathrm{~mL})$ was added to the mixture under magnetic stirring for $3 \mathrm{~h}$. The mixture solution was neutralized by a $\mathrm{HCl}$ solution $(1 \mathrm{M})$, and the precipitate was washed with distilled, deionized water and alcohol by centrifugation to remove the unreacted $\mathrm{ClCH}_{2} \mathrm{COONa}^{20,21}$.

\section{Synthesis of PEGylated graphene oxide (GO-PEG)}

The GO-COOH solution ( $1 \mathrm{mg} / \mathrm{mL}, 20 \mathrm{~mL}$, DMF) was mixed with $5 \mathrm{mg}$ of hydrophilic PEG-OH under magnetic stirring for $10 \mathrm{~min}$; then, DCC (10 mg) and DMAP (2 mg) were added to the mixture, which was stirred for $3 \mathrm{~h}$ at $60{ }^{\circ} \mathrm{C}$. Excess PEG in the as-synthesized GO-PEG sample and DMF were removed by dialysis against water using a dialysis bag (Mw cut-off: $3.5 \mathrm{kDa}$ ) for 3 days $^{22}$. 


\section{Synthesis of Ag NPs decorated on PEGylated graphene oxide nanocomposites (GO-PEG/Ag)}

The GO-PEG/Ag and HAS SD- nanocomposites were synthesized by a microwave irradiation route. In this synthesis process, GO solution $(0.25 \mathrm{mg} / \mathrm{mL}, 28 \mathrm{~mL})$ was mixed with $\mathrm{AgNO}_{3}(5 \mathrm{mM}, 5 \mathrm{~mL})$ under ultrasonication for $30 \mathrm{~min}$. Then, sodium hydroxide $(1 \mathrm{M}, 10 \mathrm{~mL})$, as a deoxygenating and reducing agent, was added to the mixture under magnetic stirring. Finally, the mixture was transferred into a microwave oven (XO-SM 100, $2.45 \mathrm{GHz}$, Galanz, Shunde, Guangdong, China). The microwave frequency was $2.45 \mathrm{GHz}$, and the input power was $200 \mathrm{~W}$ at different reaction times during the microwave irradiation process. The solution was then cooled to room temperature. The products were collected by centrifugation and washed with distilled deionized water and alcohol until the $\mathrm{pH}$ value was 7.0.

\section{Synthesis of GO-PEG/Ag-SD (HAS)}

The SD (30 mg/mL, $100 \mu \mathrm{L}, \mathrm{DMSO})$ solution was added dropwise into $10 \mathrm{~mL}$ of GO-PEG/Ag $(0.25 \mathrm{mg} / \mathrm{mL})$ under ultrasonication using a Type 60 Sonic Dismembrator (Thermo Fisher Scientific, Waltham, MA, USA). The DMSO was then completely removed by dialysis. Finally, the resulting dispersion was centrifuged for $10 \mathrm{~min}$ at $1000 \mathrm{rpm}$ to remove free SD. Subsequently, the obtained sample was dried by a freeze-drying method.

\section{Characterizations}

Scanning electron microscopy (SEM) images were obtained using a JEOL field-emission scanning electron microscope (JSM-7001 F, Japan). A model JEM-2100 instrument (JEOL, Tokyo, Japan) provided highresolution transmission electron microscopy (HRTEM) images. X-ray diffraction was performed using an XD-3 system (Purkinje General Instrument Co., Ltd., Beijing) with $\mathrm{Cu}-\mathrm{K} \alpha$ radiation $(\lambda=0.15406 \mathrm{~nm})$. The $\mathrm{UV}$-visible absorption spectra were recorded on the UV-1800 spectrophotometer in the range of $200-800 \mathrm{~nm}$, and SD had an absorption peak at $310 \mathrm{~nm}$. Fourier transform infrared (FT-IR) spectra were recorded on a TENSOR II FT-IR system (Germany) with a wavenumber range of $3800-600 \mathrm{~cm}^{-1}$ at a resolution of $4 \mathrm{~cm}^{-1}$. The antibacterial property test was investigated by a 2104 Multilabel Microplate Reader (PerkinElmer, Kentucky, USA) using MTT to signal living bacteria. Fluorescence images of bacteria were obtained using a fluorescence microscope (Nikon 80i, Japan).

\section{Antibacterial test}

The antibacterial activity of HAS nanocomposites was investigated using a gram-negative bacterium, Escherichia coli (E. coli), and gram-positive bacterium, Staphylococcus aureus (S. aureus), as two model microorganisms ${ }^{23}$. The effects of different sizes of Ag NPs to HAS SD-, HAS, and SSD on the antibacterial activity of this hybrid material and the mechanism of antibacterial activity were systematically investigated.

\section{Microdilution methods}

The hybrid materials were accurately weighed, sterilized, and then diluted with LB broth. The LB broth was used for bacterial activation, which was placed in a shaker $(170 \mathrm{rpm} / \mathrm{min})$ and incubated at $37^{\circ} \mathrm{C}$ for $12 \mathrm{~h}$. Then, $5 \mathrm{~mL}$ of the LB broth medium was mixed with $1 \%$ inoculum of activated bacteria, adding $1 \mathrm{~mL}$ of phosphate buffer, mixing evenly and diluting to a concentration of $10^{5} \mathrm{CFU} / \mathrm{mL}$ to test the antibacterial properties of $E$. coli and $S$. aureus.

To examine the minimum inhibitory concentration (MIC) of HAS, $100 \mu \mathrm{L}$ of $0.9 \%$ saline was first injected into the 96 -well plate; then, $100 \mu \mathrm{g} / \mathrm{mL}$ of the materials solution was added by pipette into the first well, and a $50 \mu \mathrm{g} /$ $\mathrm{mL}$ sample was added to the second well with the double dilution method. Finally, $10^{5} \mathrm{CFU} / \mathrm{mL}$ of bacteria was added by pipette. Seven wells had a certain amount of sample and bacteria, while $200 \mu \mathrm{L}$ of LB broth and $0.9 \%$ saline with bacteria were injected into the eleventh and twelfth wells, respectively. Through mixing, the mixture was uniform and placed in the incubator with a plastic wrap at $37^{\circ} \mathrm{C}$ to measure the OD value. Then, $20 \mu \mathrm{L}$ of MTT was added to every well. The OD value of different bacteria was determined after $4 \mathrm{~h}$. In this process, to obtain a more accurate OD value, the absorbance of materials was subtracted, and samples were measured three times for each group. The averaged OD value was useful, and the control group only had $0.9 \%$ saline added.

The living microbial cells in the abovementioned 96well plate were analyzed using the colony-forming units (CFU) method. Each culture was diluted and plated on LB-agar plates and incubated at $37^{\circ} \mathrm{C}$ for $12 \mathrm{~h}$.

\section{Diffusion method}

The diffusion method is another recognized technique to determine the in vitro susceptibility of bacteria to various drugs. The disk concentration $(10 \mathrm{mg} /$ disk $)$ was based on preliminary experiments in which blank disks were impregnated with $10 \mathrm{~mL}$ of suspension containing $1 \mathrm{mg} / \mathrm{mL}$ drug. Specifically, $\mathrm{MH}$ agar plates were inoculated with a bacterial suspension of $10^{5} \mathrm{CFU} / \mathrm{mL}$ by swabbing the agar surface. After the plates were dry, sterile paper disks containing the corresponding drug, HAS, HAS SD- and SSD were placed on the agar surface. The diameters (in $\mathrm{mm}$ ) of the zone of complete inhibition were determined after $25 \mathrm{~h}$ of incubation at $37^{\circ} \mathrm{C}$. 


\section{Fluorescence-based live/dead bacterial assay}

E. coli and S. aureus cells were inoculated in fresh LB broth at $10^{5} \mathrm{CFU} / \mathrm{mL}$ with HAS $(50 \mu \mathrm{g} / \mathrm{mL})$. After growth for $1 \mathrm{~h}$ at $37^{\circ} \mathrm{C}$ in the shaking incubator, the bacteria were collected by centrifugation, stained with propidium iodide (PI, $1 \mu \mathrm{g} / \mathrm{mL}$ ) for $15 \mathrm{~min}$, counterstained with 4'-6-diamidino-2-phenylindole (DAPI, $5 \mu \mathrm{g} / \mathrm{mL}$ ) for $5 \mathrm{~min}$ in the dark, and then imaged using a Nikon 80i fluorescence microscope.

\section{Results and discussion}

\section{Synthesis and characterization of HAS}

The modified Hummers method was applied to prepare the graphite oxide ${ }^{24}$; GO sheets with carboxylic groups, epoxide groups, and phenol hydroxyl at its edges and basal plane were produced by the oxidation process ${ }^{25}$. To improve the stability of the material in water, hydroxyl groups were converted to carboxylic groups via carboxylation, which could provide more reactive sites for covalent modifications such as the esterification of carboxyl groups ${ }^{26}$. The existence of oxygen-containing groups on the surface of GO was examined by FT-IR spectroscopy (Fig. 1). As shown in Fig. 1a, the presence of an absorption band at approximately $1625 \mathrm{~cm}^{-1}$ corresponds to the $\mathrm{C}=\mathrm{C}$ bonds of the aromatic rings of the GO carbon skeleton structure. The absorption band at approximately $3454 \mathrm{~cm}^{-1}$ is from -OH groups, which may come from distilled water and hydroxyl groups. The vibrational peaks at approximately 1078, 1384, and $1720 \mathrm{~cm}^{-1}$ are assigned to the $\mathrm{C}=\mathrm{O}, \mathrm{C}-\mathrm{OH}$, and $\mathrm{C}=\mathrm{O}$ stretching vibrations of $\mathrm{COOH}$ groups, respectively.

After the carboxylation reaction, the peak at $1720 \mathrm{~cm}^{-1}$ in the FT-IR spectrum was stronger than that in the spectrum of GO (Fig. 1a). This evidence distinctly demonstrated that the initial hydroxyl groups on the surface of the GO sheets were converted to carboxylic groups. After conjugation with PEG following the esterification reaction, a new signal attributed to ester bonds (Fig. 1b) appeared at $1735 \mathrm{~cm}^{-1}$ in the FT-IR spectrum. The result directly confirmed that PEG was successfully conjugated to GO.

The morphology of GO, GO-COOH, and GO-PEG was visualized by SEM, as shown in Fig. 2. The GO sheets were loosely stacked with some wrinkles, and the surface was smooth (Fig. 2a). The surface appearances of carboxyl-functionalized GO sheets (Fig. 2b) and PEGylated GO sheets (Fig. 2c) were similar to those of GO sheets but with more wrinkles.
A

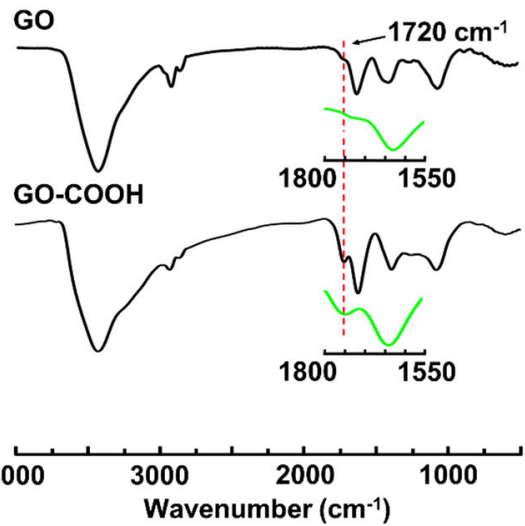

B

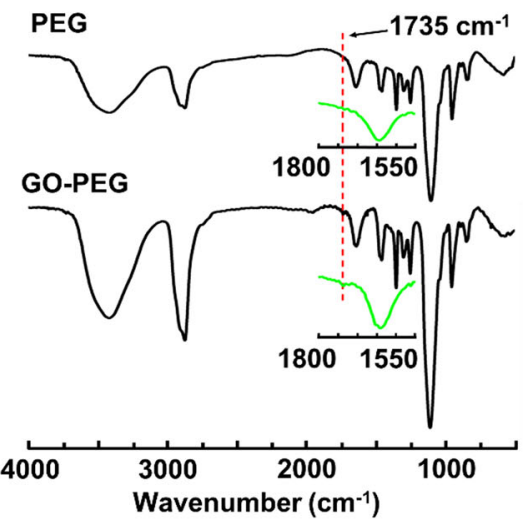

Fig. 1 FT-IR spectra of GO, GO-COOH a, and PEG, GO-PEG $\mathbf{b}$
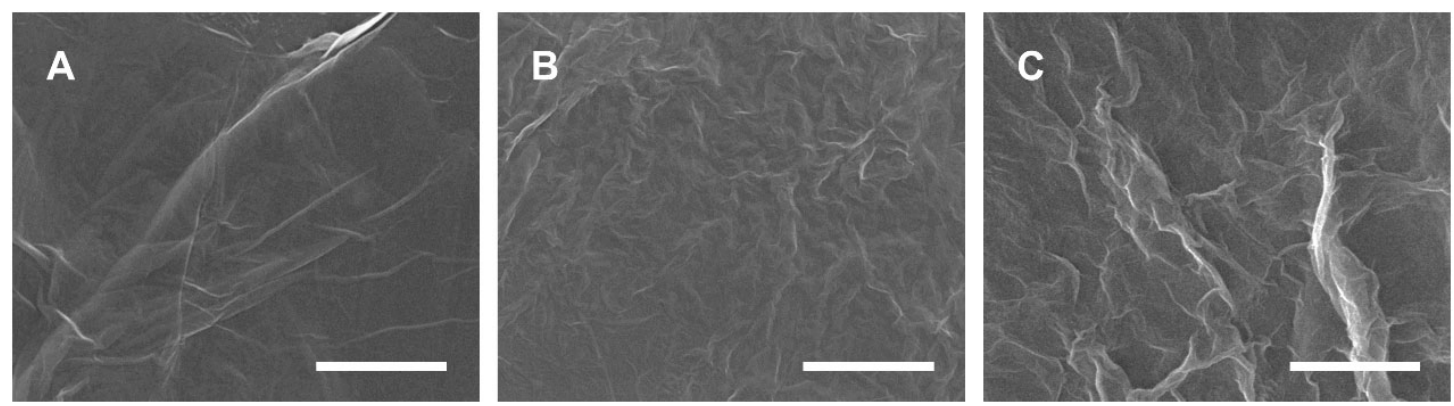

Fig. 2 SEM images of a GO, b GO-COOH, c GO-PEG. Scale bars are $1 \mu \mathrm{m}$. 


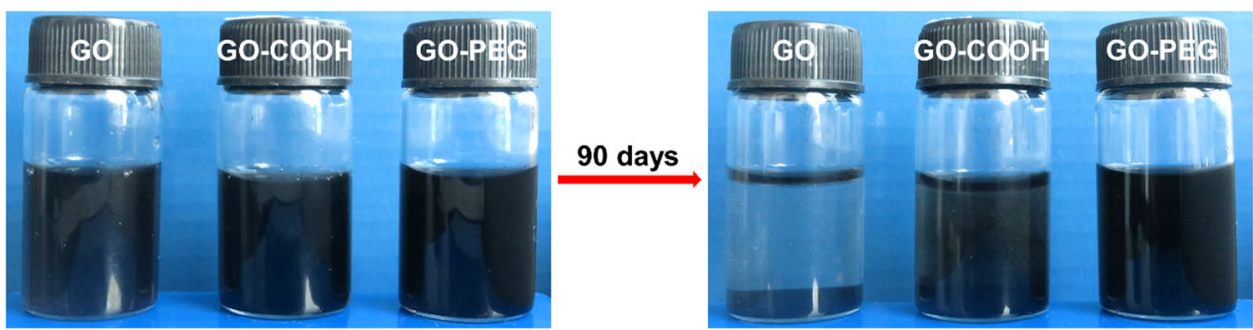

Fig. 3 Water stability of GO, GO-COOH, and GO-PEG for 90 days.
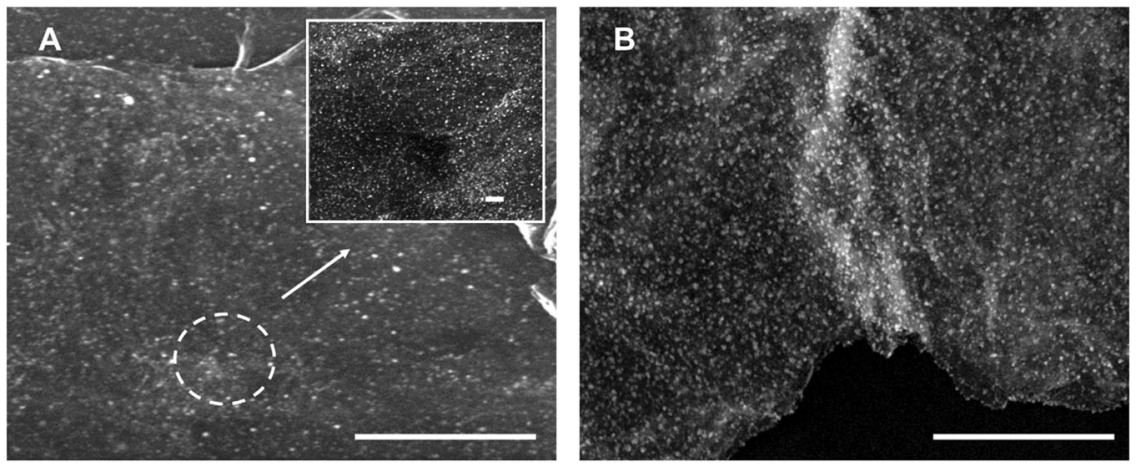

Fig. 4 SEM images and size distributions of GO-PEG/Ag at different microwave irradiation times: a 200 W, 1 min (Ag NPs 8 nm, GO-Ag1); the insets are

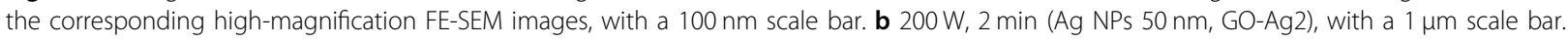

The stability of GO, GO-COOH, and GO-PEG was tested in aqueous media for 96 days (Fig. 3). GO-PEG was found to be stable in water without precipitation; PEGylated graphene oxide exhibited significantly improved water stability. The unfunctionalized GO, which was suspended in water via a small number of hydrophilic groups, such as carboxylic groups, could not provide sufficient buoyancy force. When hydrophilic PEG-OH was introduced into the carboxylated graphene oxide $(\mathrm{GO}-\mathrm{COOH})$ as GO-PEG, the number of hydrophilic groups was significantly increased, which could provide enough buoyancy force to keep the GO sheets stable in aqueous solution.

The preparation of GO-PEG/Ag by a facile synthesis method via microwave irradiation is a rapid and green route. The size of Ag NPs can be easily controlled by adjusting the microwave time.

SEM was employed to characterize the morphology and microstructure of the graphene-based hybrid materials to verify the effective anchoring of Ag NPs onto large GO-PEG sheets. As shown in Fig. 4a, GO-PEG/Ag was synthesized at $200 \mathrm{~W}$ for $1 \mathrm{~min}$, and Ag NPs with an average diameter of $8 \mathrm{~nm}$ were embedded on the GOPEG sheets. When the time was changed to $2 \mathrm{~min}$, the size of the Ag NPs was $\sim 50 \mathrm{~nm}$ (Fig. 4b). The Ag NPs did not agglomerate.
TEM, HRTEM, and XRD images of the GO-PEG/Ag composite (200 W, $1 \mathrm{~min})$ are shown in Fig. 5. Ag NPs with a size of $8 \mathrm{~nm}$ were highly monodispersed on the GO-PEG sheet (Fig. 5a). The TEM image shows that Ag NPs were embedded on the surface of the GO-PEG sheets. The HRTEM (Fig. 5a) lattice distance was $0.236 \mathrm{~nm}$, indicating a lattice plane of $\mathrm{Ag}(111)^{27}$, which is in accordance with the XRD (Fig. 5b) profiles of the GO and GO-PEG/Ag samples (200 W, $1 \mathrm{~min}$ ). The (001) diffraction peak of graphene oxide at $10.8^{\circ}$ was retained after microwave irradiation, indicating that the hybrid materials still have a large number of functional groups on the GO surface. The face-centered cubic Ag crystal planes of (111), (200), (220), and (311) correspond to the peaks at $2 \theta=38.2^{\circ}, 44.2^{\circ}, 64.5^{\circ}$, and $77.3^{\circ}$, respectively, proving that the metallic Ag NPs were formed under the microwave irradiation power applied for $1 \mathrm{~min}$ at $200 \mathrm{~W}$.

As shown in Fig. 6a, the FT-IR spectrum of GO-PEG/ Ag-SD was different from that of GO-PEG. New signals appeared at $\quad 3424 \mathrm{~cm}^{-1}, \quad 3356 \mathrm{~cm}^{-1}, \quad 2874 \mathrm{~cm}^{-1}$, $2136 \mathrm{~cm}^{-1}, 1654 \mathrm{~cm}^{-1}, 1156 \mathrm{~cm}^{-1}$ and $1097 \mathrm{~cm}^{-1}$ in the FT-IR spectrum of GO-PEG/Ag-SD, which were attributed to $\mathrm{SD}$ and indicated the successful loading of SD onto GO-PEG/Ag.

The drug loading ability of GO-PEG/Ag was verified by UV-vis spectroscopy. Figure S1 shows the standard 

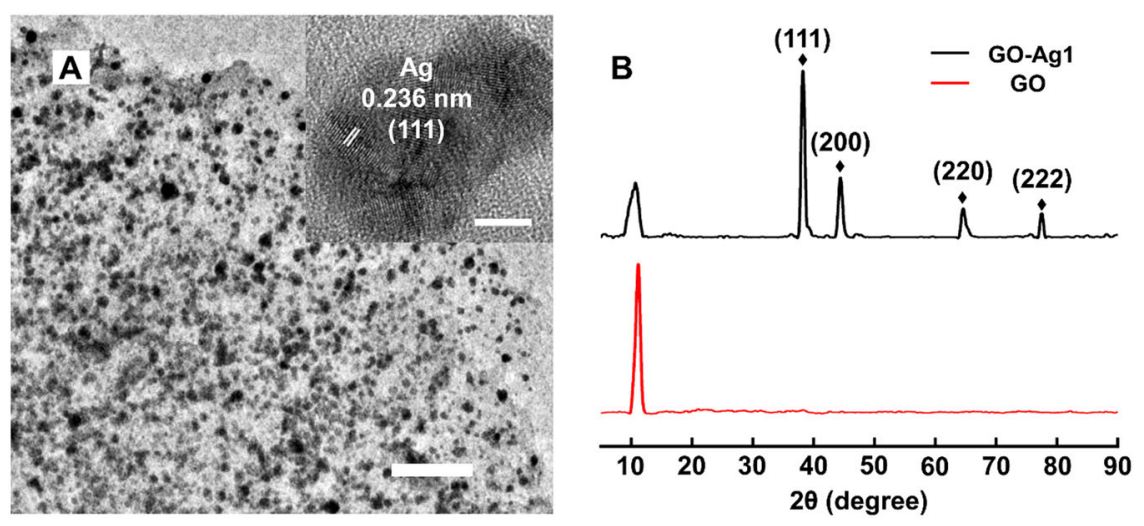

Fig. 5 a TEM images of GO-Ag1 with a $100 \mathrm{~nm}$ scale bar and the corresponding high-magnification HR-TEM images insets with a $5 \mathrm{~nm}$ scale bar. b XRD patterns of GO and GO-Ag1.
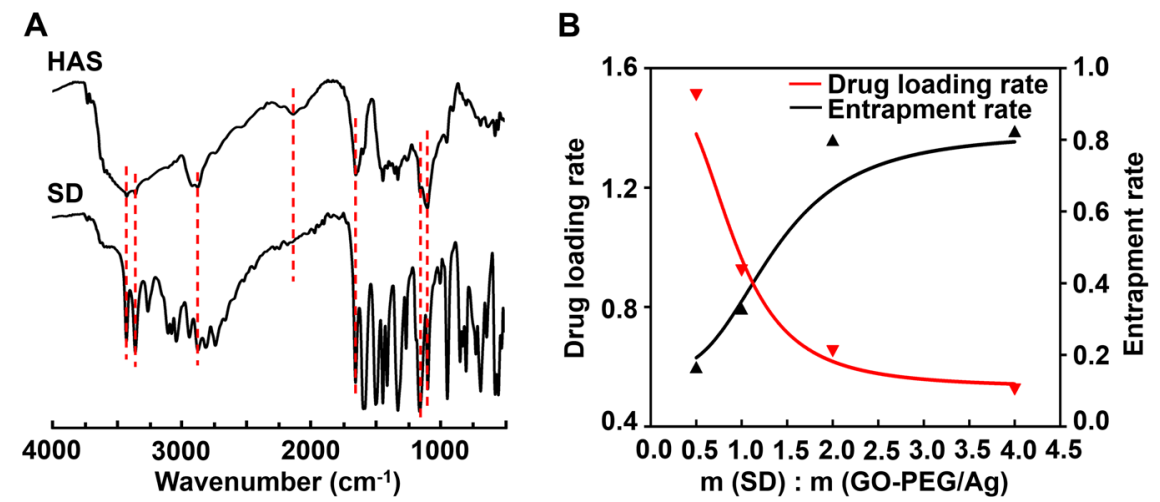

Fig. 6 a FT-IR spectra of SD and HAS. b UV-vis spectra of drug loading and entrapment rate curves of HAS.

UV-vis curve of the SD solution, with an absorption wavelength at $310 \mathrm{~nm}$. With an increase in the SD: GOPEG ratio (0.5:1, 1:1, 2:1, and 4:1, by weight), the drug loading content increased $(59.24 \%, 78.83 \%, 135.26 \%$, and $138.24 \%$, respectively) and the entrapment rate decreased (93.00\%, 44.08\%, 21.56\%, and 10.88\%), as shown in Fig. 6b; the drug loading rate decreased and then gradually leveled off, but the entrapment rate was opposite to the drug loading rate. The 2:1 SD:GO-PEG ratio shows the maximal drug loading and best entrapment rate, and this ratio was chosen to synthesize the GO-PEG/Ag-SD composite.

\section{In vitro evaluation of the antibacterial activity The effect of Ag NP size on the antibacterial activity}

Ag NPs have been extensively explored for their excellent antibacterial activity ${ }^{28}$ In this study, Ag NPs of different sizes were synthesized by changing the microwave irradiation time. The smaller Ag NPs GO-Ag1 (8 nm) showed better antibacterial activity than the larger $\mathrm{Ag}$ NPs GO-Ag2 $(50 \mathrm{~nm}$ ) (Fig. 7a), with $6.25 \mu \mathrm{g} / \mathrm{mL}$ and $12.5 \mu \mathrm{g} / \mathrm{mL}$ MIC values, respectively (Fig. $7 \mathrm{~b}$ ). This trend is consistent with previous research ${ }^{15,29}$. Based on this result, GO-Ag1 was chosen as the precursor to load SD.

\section{Antibacterial activity of HAS}

The antibacterial activity of HAS against $E$. coli was determined by MTT assay using HAS SD- and SSD as control groups. HAS showed better antibacterial activity (Fig. 8a), with almost complete inhibition of the growth of E. coli. As shown in Fig. 8b, c, the HAS MIC was $\sim 0.78 \mu \mathrm{g} /$ $\mathrm{mL}$ for $E$. coli, showing over 3 times improved antibacterial activity compared to that of HAS SD- $(3.12 \mu \mathrm{g} /$ $\mathrm{mL})$. The results showed that HAS presents the best synergistic antibacterial activity towards E. coli.

The paper disk method was also studied to further compare the antibacterial activity of HAS with that of HAS SD- and SSD (Fig. 9). Compared to that of HAS SDand SSD, a larger inhibition zone diameter against $E$. coli was observed for the HAS, indicating that it has the best antimicrobial activity.

The antibacterial activity of HAS, HAS SD- and SSD towards the gram-positive bacterium $S$. aureus was also 

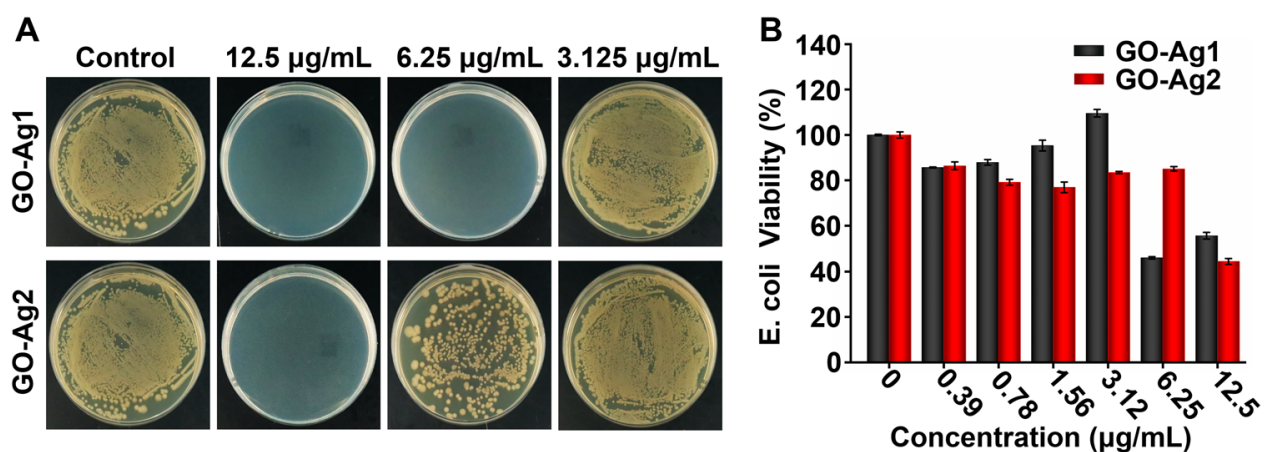

Fig. 7 Antibacterial activity of GO-PEG/Ag. a Bacterial colony formation of E. coli under different treatments. b Bacterial cell viability of E. coli with different sizes of Ag NPs.
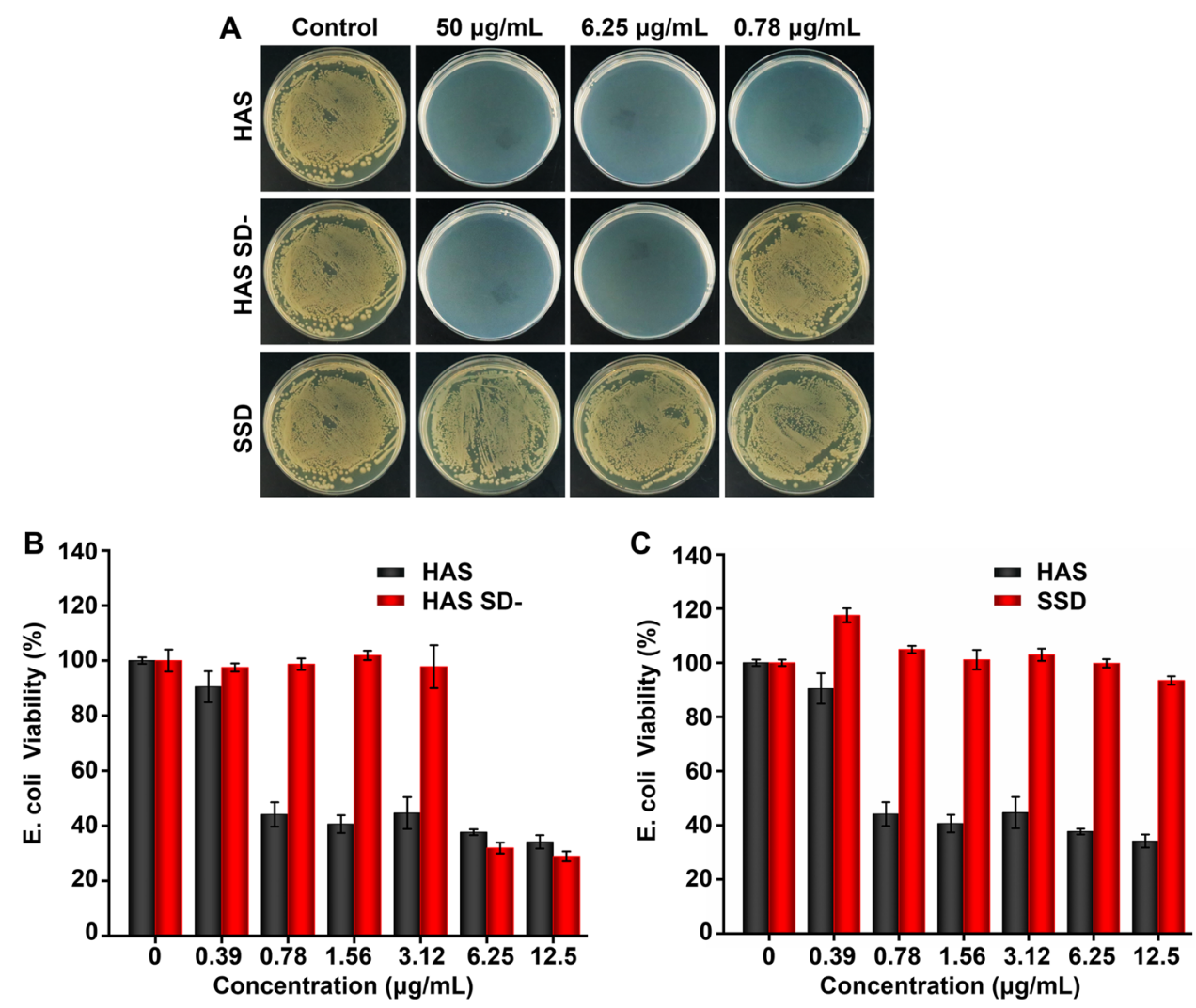

Fig. 8 Antibacterial activity of HAS. a Bacterial colony formation of $E$. coli treated with different materials. $\mathbf{b}$ Bacterial cell viability of $E$. coli with HAS and HAS SD-. c Bacterial cell viability of E. coli with HAS and SSD.

explored. As shown in Figs. S2a and S3a, the results of the bacterial colony counts showed that there was no significant difference in antibacterial activity between HAS and HAS SD-. Figs. S2b and S3b show the same antibacterial activity results for HAS and HAS SD-, and the MICs were both $6.25 \mu \mathrm{g} / \mathrm{mL}$. The paper disk method results presented the same trend (Fig. S4).

\section{Antimicrobial mechanism of HAS}

To study the antibacterial mechanism of HAS, two fluorescent nucleic acid dyes, DAPI and PI, were employed to stain the DNA of bacteria. DAPI labels both live and dead cells, while PI can only penetrate cells with compromised or damaged membranes ${ }^{30}$, which can be used to test whether the bacteria are live (blue) or dead 
(red). As shown in Fig. 10, the E. coli and S. aureus cells were almost completely stained by PI, which shows damaged cell walls and cell membranes and/or mass cell death after treatment with HAS $(50 \mu \mathrm{g} / \mathrm{mL})$ for $1 \mathrm{~h}$, and the efficiency of sterilization was above $98 \%$, showing efficient and fast antibacterial activity.

Scanning electron microscopywas used to study the interactions between HAS and bacteria. As shown in Fig. 11, HAS served as a net to wrap E. coli and S. aureus in the treated samples, which limited bacteria movement. The large size of HAS ensured that it could trap or wrap many bacteria and kill or inhibit bacterial growth. The surfaces of $E$. coli and $S$. aureus were obviously wrinkled and damaged. Cells were observed to be leaking cytoplasm, and bacteria were in contact with the Ag NPs.

The above results indicated that the HAS presented excellent antibacterial activity against $E$. coli. The hybrid system, which consisted of GO-PEG, Ag NPs, and SD, exhibited superior synergistic antimicrobial activity via

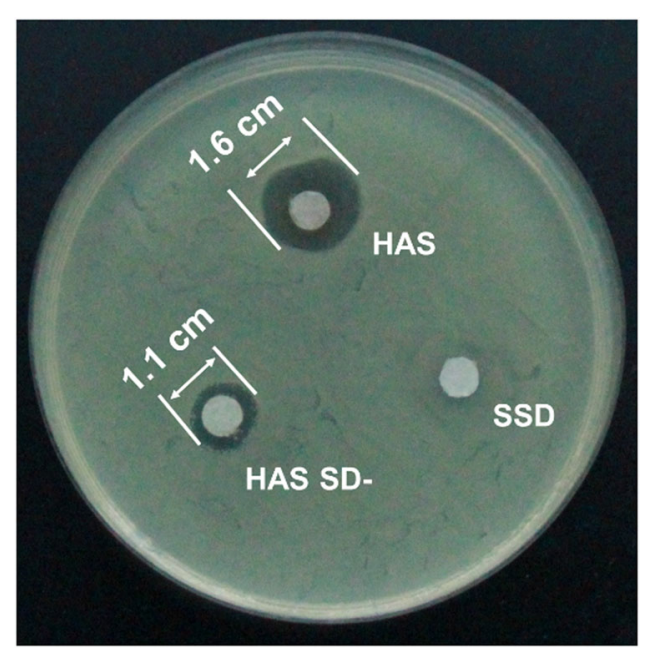

Fig. 9 Antibacterial activity of HAS by the disk-diffusion method. E. coli with HAS, HAS SD- and SSD. three effects (Fig. 12b): (1) The capping effect of graphene oxide. Adsorption of the antibacterial system to the bacteria is the most important step in killing or inhibiting bacterial growth. As shown in Fig. 12a, bacteria were wrapped or trapped by the thin sheets of HAS via intermolecular forces ${ }^{31}$. The outer membrane of bacteria contains sugars, phosphates, and lipids, which can form hydrogen bonds with HAS and subsequently form agglomerates. HAS adsorption on the bacteria served two antibacterial functions: (i) it significantly increased the concentration of Ag NPs and SD around the bacteria and (ii) blocked nutrient transport. The entry of nutrients into the cell and the delivery of excreta out of the cell are critical for cell and bacterial growth ${ }^{32}$. It has been demonstrated that it is hard for nutrients to pass across GO-based hybrid materials, which indicates that bacterial nutritional supplementation and metabolism will be inhibited once bacteria are trapped by GO-based hybrid materials $^{33,34}$. (2) The puncture effect of Ag NPs. Small Ag NPs $(8 \mathrm{~nm})$ with positive surface potential can easily be adsorbed onto the bacterial surface, which can affect the permeability of the bacterial cell membranes and rupture the membranes ${ }^{35,36}$. (3) The inhibitory effect of SD. SD could directly inhibit nucleic acid synthesis in bacterial cells. SD has a similar chemical structure to that of para-aminobenzoic acid, which is a necessary substance for adsorbing folic acid and can inhibit bacterial adsorption of folic acid, thus further inhibiting the growth of bacteria. Additionally, SD can weaken the E. coli cell wall to some extent ${ }^{37,38}$ and further enhance the cell membrane rupture ability of Ag NPs, highlighting the significant synergistic antibacterial effect of Ag NPs and SD.

\section{Conclusion}

A novel 2D hybrid-material-based antibacterial system was developed via a facile, rapid, microwave-assisted green route and efficient antibacterial drug loading. The HAS possessed superior water phase stability through surface hydrophilic modification. The high specific

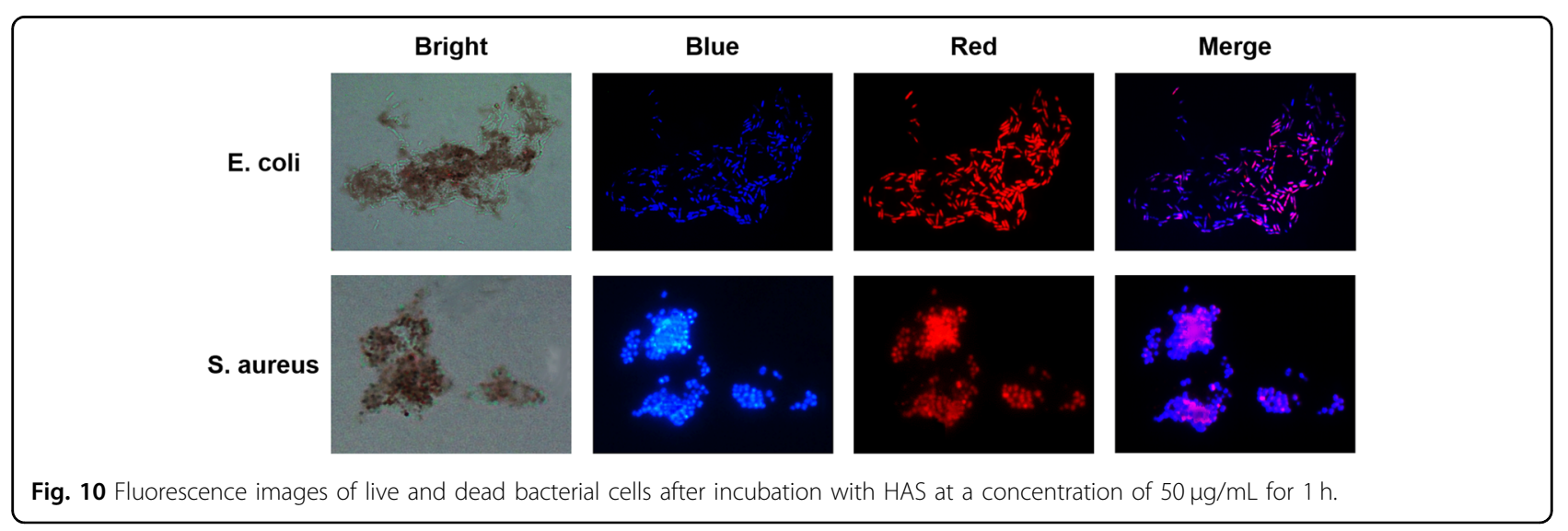



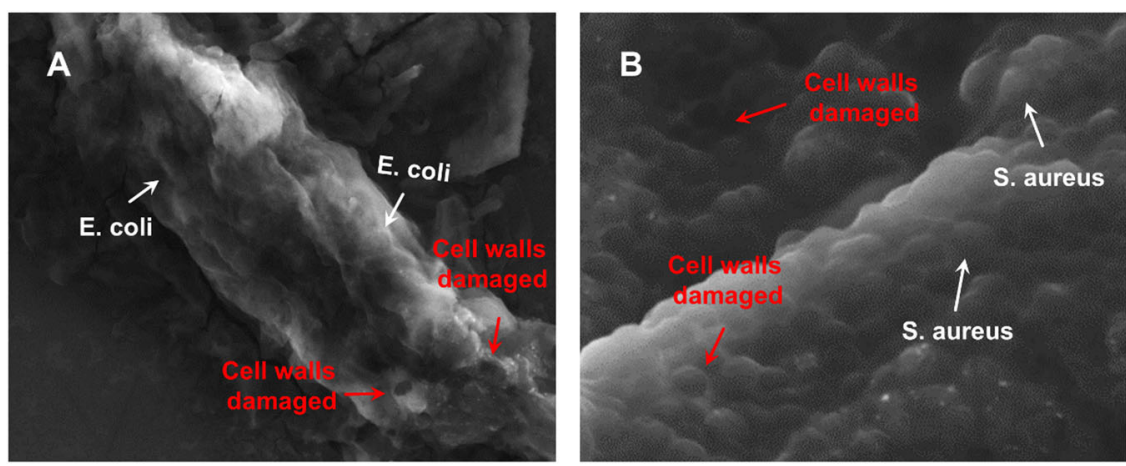

Fig. 11 SEM images of E. coli cells $\mathbf{a}$ and S. aureus cells $\mathbf{b}$ treated with $\mathrm{HAS}(50 \mu \mathrm{g} / \mathrm{mL})$ for $1 \mathrm{~h}$.

A
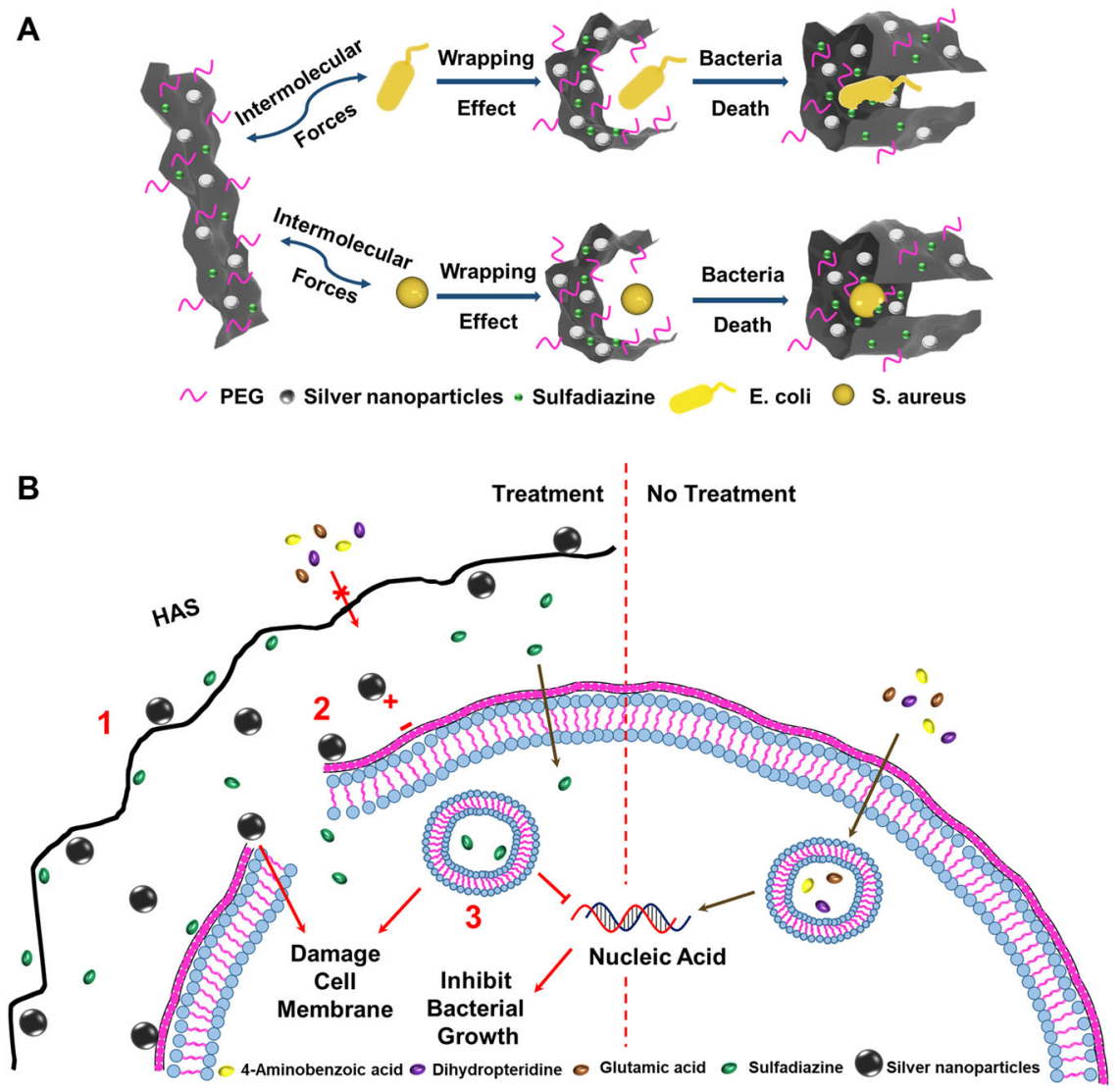

Fig. 12 Schematic illustration of the interaction between HAS and microorganisms $\mathbf{a}$ and the synergistic antibacterial mechanism of HAS $\mathbf{b}$. The antibacterial mechanism could be divided into three parts (Fig. 12B): (1) The capping effect of graphene oxide via intermolecular forces has two antibacterial functions: (i) significantly enhancing the concentration of Ag NPs and SD around bacteria and (ii) blocking nutrient transport; (2) The puncture effect of Ag NPs; and (3) The inhibitory effect of SD, which could directly inhibit nucleic acid synthesis in bacterial cells and could weaken the E. coli cell wall. In this study, a novel hybrid antibacterial system (HAS) based on graphene oxide (GO) 2D materials for co-delivery of silver nanoparticles (Ag NPs) and sulfadiazine (SD) was fabricated. The antibacterial activity of HAS was enhanced over 3 times due to triple synergy: capping effect of GO, puncture effect of Ag NPs, and inhibitory effect of SD. This study provides new insights into the design and fabrication of antibiosis systems and multifunctional materials and broadens the biomedical applications of 2D materials.

surface area GO was utilized to efficiently load antibacterial Ag NPs and SD. The HAS revealed excellent antibacterial activity with a remarkably low MIC dosage
$(0.78 \mu \mathrm{g} / \mathrm{mL})$, fast sterilization and superior efficiency. These properties are attributed to the excellent synergistic antibacterial effect of GO, Ag NPs, and SD: the capping 
effect of GO, puncture effect of Ag NPs, and inhibitory effect of SD. This study provides new insights into the design and fabrication of surface-modified GO and carbon materials ${ }^{39-43}$ and their $2 \mathrm{D}$ hybrid multifunctional materials for advanced applications including biomedi$\mathrm{cal}^{44}$ especially antibacterial applications, broadening the design and application scope of carbon and 2D materials.

\section{Acknowledgements}

This research was supported by the National Natural Science Foundation of China (81600775, 51602287, and 21504082), the National Key R\&D Program of China (2016YFE0117100), and the Medical Science and Technology Program of Henan Province (2018020398 and SB201902026).

\section{Author details}

'Zhengzhou University People's Hospital, Henan Provincial People's Hospital, Zhengzhou 450003, PR China. ${ }^{2}$ School of Materials Science and Engineering, Zhengzhou University, Zhengzhou 450001, PR China. ${ }^{3}$ Henan Key Laboratory of Aeronautical Material and Application Technology, Zhengzhou University of Aeronautics, Zhengzhou 450015, PR China. ${ }^{4}$ John A. Paulson School of Engineering and Applied Sciences, Harvard University, Cambridge, MA 02138, USA

\section{Conflict of interest}

The authors declare that they have no conflict of interest.

\section{Publisher's note}

Springer Nature remains neutral with regard to jurisdictional claims in published maps and institutional affiliations.

Supplementary information is available for this paper at https://doi.org/ 10.1038/s41427-020-0195-x.

Received: 25 October 2019 Revised: 4 December 2019 Accepted: 10 December 2019.

Published online: 31 January 2020

\section{References}

1. Zhang, Y., Tan, Y.-W., Stormer, H. L. \& Kim, P. Experimental observation of the quantum Hall effect and Berry's phase in graphene. Nature 438, 201-204 (2005).

2. Geim, A. K. Graphene: status and prospects. Science 324, 1530-1534 (2009).

3. Luk'Yanchuk, I. A. \& Yakov, K. Dirac and normal fermions in graphite and graphene: implications of the quantum Hall effect. Phys. Rev. Lett. 97, 256801 (2006).

4. Meyer, J. C. et al. The structure of suspended graphene sheets. Nature $\mathbf{4 4 6}$, 60-63 (2007).

5. Novoselov, K. S. et al. Electric field effect in atomically thin carbon films. Science 306, 666-669 (2004)

6. Yang, K. et al. Graphene in mice: ultrahigh in vivo tumor uptake and efficient photothermal therapy. Nano Lett. 10, 3318 (2010).

7. Ramanathan, T. et al. Functionalized graphene sheets for polymer nanocomposites. Nat. Nanotechnol. 3, 327-331 (2008)

8. Dong, M., Lin, J., Chen, Y., Wei, X. \& Zhang, L. M. In situ gelation and sustained release of an antitumor drug by graphene oxide nanosheets. Carbon 50, 3001-3007 (2012).

9. Wang, F. et al. Imaging dendrimer-grafted graphene oxide mediated anti-miR21 delivery with an activatable Luciferase reporter. ACS Appl. Mater. Interfaces $\mathbf{8}$, 9014-9021 (2016)

10. Omid, A. \& Elham, G. Toxicity of graphene and graphene oxide nanowalls against bacteria. ACS Nano 4, 5731-5736 (2010).

11. Fadeel, B. et al. Safety assessment of graphene-based materials: focus on human health and the environment. ACS Nano 12, 10582-10620 (2018).

12. Xin Q., et al. Antibacterial carbon-based nanomaterials. Adv. Mater. 31, 1804838 (2018).

13. Tang, J. et al. Graphene oxide-silver nanocomposite as a highly effective antibacterial agent with species-specific mechanisms. ACS Appl. Mater. Interfaces 5, 3867-3874 (2013).
14. Shaobin, L. et al. Antibacterial activity of graphite, graphite oxide, graphene oxide, and reduced graphene oxide: membrane and oxidative stress. ACS Nano 5, 6971-6980 (2011).

15. Li, Y. et al. Rational Design of Silver Gradient for Studying Size Effect of Silver Nanoparticles on Contact Killing. ACS Biomater. Sci. Eng. 5, 425-431 (2018).

16. Panáček, A. et al. Bacterial resistance to silver nanoparticles and how to overcome it. Nat. Nanotechnol. 13, 65 (2018).

17. Jin, L. et al. Fabrication, mechanical properties, and biocompatibility of reduced graphene oxide-reinforced nanofiber mats. RSC Adv. 4, 35035-35041 (2014).

18. Kumar, S. \& Chatterjee, K. Comprehensive review on the use of graphenebased substrates for regenerative medicine and biomedical devices. ACS Appl. Mater. Interfaces 8, 26431-26457 (2016).

19. Hummers, W. S. \& Offeman, R. E. Preparation of graphitic oxide. J. Am. Chem Soc. 80, 1339 (1958)

20. Yang, C., Hao, S.-J., Dai, S.-L. \& Zhang, X.-Y. Nanocomposites of poly(vinylidene fluoride) - Controllable hydroxylated/carboxylated graphene with enhanced dielectric performance for large energy density capacitor. Carbon 117 301-312 (2017).

21. Liu, G. et al. Transferrin modified graphene oxide for glioma-targeted drug delivery: in vitro and in vivo evaluations. ACS Appl. Mater. Interfaces $\mathbf{5}$ 6909-6914 (2013).

22. Xu, Z. et al. Covalent functionalization of graphene oxide with biocompatible poly(ethylene glycol) for delivery of paclitaxel. ACS Appl. Mater. Interfaces 6 17268-17276 (2014).

23. Osborn, M. J. Structure and biosynthesis of the bacterial cell wall. Annu. Rev. Biochem. 38, 501-538 (1969).

24. Fang, M., Wang, K., Lu, H., Yang, Y. \& Nutt, S. Covalent polymer functionalization of graphene nanosheets and mechanical properties of composites. J. Mater. Chem. 19, 7098-7105 (2009).

25. Yang, X., Zhang, X., Liu, Z., Ma, Y., Yi, H. \& Chen, Y. High-efficiency loading and controlled release of doxorubicin hydrochloride on graphene oxide. J. Phys. Chem. C 112, 17554-17558 (2008).

26. Yang, $H$. et al. Covalent functionalization of polydisperse chemically-converted graphene sheets with amine-terminated ionic liquid. Chem. Commun. 45, 3880-3882 (2009)

27. Shao, W. et al. Preparation, characterization, and antibacterial activity of silver nanoparticle-decorated graphene oxide nanocomposite. ACS Appl. Mater. Interfaces 7, 6966-6973 (2015).

28. Du, Y. et al. Preparation of versatile yolk-shell nanoparticles with a precious metal yolk and a microporous polymer shell for high-performance catalysts and antibacterial agents. Polymer 137, 195-200 (2018).

29. Eby, D. M., Luckarift, H. R. \& Johnson, G. R. Hybrid antimicrobial enzyme and silver nanoparticle coatings for medical instruments. ACS Appl. Mater. Interfaces 1, 1553 (2009).

30. Vergaro, V. et al. Drug-loaded polyelectrolyte microcapsules for sustained targeting of cancer cells. Adv. Drug Deliv. Rev. 63, 847-864 (2011).

31. Akhavan, O., Ghaderi, E. \& Esfandiar, A. Wrapping bacteria by graphene nanosheets for isolation from environment, reactivation by sonication, and inactivation by near-infrared irradiation. J. Phys. Chem. B 115, 6279-6288 (2011).

32. Li, Z. et al. Biomimicry, biomineralization, and bioregeneration of bone using advanced three-dimensional fibrous hydroxyapatite scaffold. Mater. Today Adv. 3, 100014 (2019).

33. Katoch, J. et al. Structure of a peptide adsorbed on graphene and graphite. Nano Lett. 12, 2342-2346 (2012).

34. Lei, $\mathrm{H}$. et al. Adsorption of double-stranded DNA to graphene oxide preventing enzymatic digestion. Nanoscale 3, 3888-3892 (2011).

35. Yang, L. \& Ning, G. Thermodynamics of charged nanoparticle adsorption on charge-neutral membranes: a simulation study. J. Phys. Chem. B 114 2749-2754 (2010)

36. Le Ouay, B. \& Stellacci, F. Antibacterial activity of silver nanoparticles: a surface science insight. Nano today 10, 339-354 (2015).

37. McMahon, S. et al. Poly(ethylene glycol)-based hyperbranched polymer from RAFT and its application as a silver-sulfadiazine-loaded antibacterial hydrogel in wound care. ACS Appl. Mater. Interfaces 8, 26648-26656 (2016).

38. Coward, J. E., Carr, H. S. \& Rosenkranz, H. S. Silver sulfadiazine: effect on the ultrastructure of Pseudomonas aeruginosa. Antimicrobial Agents Chemother. 3 621-624 (1973)

39. Xie, Y. et al. Synergistic cobalt sulfide/eggshell membrane carbon electrode ACS Appl. Mater. interfaces 11, 32244-32250 (2019). 
40. Meng, J. et al. Identification of phase control of carbon-confined $\mathrm{Nb}_{2} \mathrm{O}_{5}$ nanoparticles toward high-performance lithium storage advanced energy. Materials 9, 1802695 (2019).

41. Du, Y. et al. In-situ preparation of porous carbon nanosheets loaded with metal chalcogenides for a superior oxygen evolution reaction. Carbon 149, 144-151 (2019)

42. Wu, J. et al. Porous polymers as multifunctional material platforms toward taskspecific applications. Adv. Mater. 31, 1802922 (2019).
43. Zheng B., Lin X., Zhang X., Wu D. \& Matyjaszewski K. Emerging Functional Porous Polymeric and Carbonaceous Materials for Environmental Treatment and Energy Storage Advanced Functional Materials. https://doi.org/10.1002/ adfm.201907006 (2019).

44. Gao D., et al. Multifunctional phototheranostic nanomedicine for cancer imaging and treatment. Materials Today Bio https://doi.org/10.1016/j. mtbio.2019.100035 (2019). 